\title{
Effect of load triaxiality on polymorphic transitions in zinc oxide
}

\author{
A.J. Kulkarni a , K. Sarasamak b, J. Wang ${ }^{\text {c }}$, F.J. Ke ${ }^{\text {c,d }}$, \\ S. Limpijumnong ${ }^{b}$, M. Zhou ${ }^{a, *}$ \\ ${ }^{a}$ The George W. Woodruff School of Mechanical Engineering, Georgia Institute of Technology, Atlanta, GA 30332-0405, USA \\ ${ }^{\mathrm{b}}$ School of Physics, Suranaree University of Technology and National Synchrotron Research Center, \\ Nakhon Ratchasima 30000, Thailand \\ ${ }^{\mathrm{c}}$ Department of Physics, Beihang University, Beijing 100083, China \\ d Institute of Mechanics, Chinese Academy of Sciences, Beijing 100080, China
}

Received 31 August 2007

Available online 21 September 2007

\begin{abstract}
Molecular dynamics (MD) simulations and first-principles calculations are carried out to analyze the stability of both newly discovered and previously known phases of $\mathrm{ZnO}$ under loading of various triaxialities. The analysis focuses on a graphite-like phase (HX) and a body-centered-tetragonal phase (BCT-4) that were observed recently in [01 $\overline{1} 0]$ - and [0001]-oriented nanowires respectively under uniaxial tensile loading as well as the natural state of wurtzite (WZ) and the rocksalt (RS) phase which exists under hydrostatic pressure loading. Equilibrium critical stresses for the transformations are obtained. The $\mathrm{WZ} \rightarrow \mathrm{HX}$ transformation is found to be energetically favorable above a critical tensile stress of $10 \mathrm{GPa}$ in [0110] nanowires. The BCT-4 phase can be stabilized at tensile stresses above $7 \mathrm{GPa}$ in [0001] nanowires. The RS phase is stable at hydrostatic pressures above $8.2 \mathrm{GPa}$. The identification and characterization of these phase transformations reveal a more extensive polymorphism of $\mathrm{ZnO}$ than previously known. A crystalline structure-load triaxiality map is developed to summarize the new understanding.
\end{abstract}

(c) 2007 Elsevier Ltd. All rights reserved.

Keywords: Phase transformations; Load triaxiality; Zinc oxide; Molecular dynamics; Density functional theory

\section{Introduction}

Polymorphic transitions occur in materials with non-convex free energy landscapes or materials that display multiple local minima along with a global minimum under ambient conditions. The global minimum in free energy corresponds to a stable crystalline structure and is the natural state of the material. Each local minimum, on the other hand, represents a metastable lattice structure that the material can assume under external stimuli. Traditionally, external loading and temperature changes are used to transform materials from their stable structures to metastable states. Stress-induced phase transformations are widely observed in

\footnotetext{
* Corresponding author. Tel.: +1 404894 3294; fax: +1 4048940186.

E-mail address: min.zhou@gatech.edu (M. Zhou).
} 
groups IV, III-V and II-VI materials including $\mathrm{ZnO}$, which have been predominantly studied through compressive loading (Mujica et al., 2003). Having a parent wurtzite (WZ, P6 $6_{3} m c$ space group) structure, these materials transform to a rocksalt (RS, $F m \overline{3} m$ space group) structure under high hydrostatic pressures. The recent fabrication of defect-free, single-crystalline nanowires, nanobelts and nanorings of $\mathrm{ZnO}$ (IIB-VIA), $\mathrm{GaN}$ (IIIA-VA) and CdSe (IIB-VIA) necessitates the analyses of responses to loading of various triaxialities, including bending and uniaxial tension since these materials have slender quasi one-dimensional geometries and are capable of undergoing significant elongations (Diao et al., 2004; Kulkarni et al., 2005; Liang and Zhou, 2006).

There are three hitherto known polymorphs of $\mathrm{ZnO}$, including WZ, RS and zinc blende ( $\mathrm{ZB}, F \overline{4} 3 m)$ (Ozgur et al., 2005). WZ is the natural state under ambient conditions. RS occurs under high hydrostatic pressures. ZB can only be grown on certain crystalline surfaces of cubic crystals. So far, the existence of polymorphs other than WZ, ZB and RS at various loading triaxialities has not been extensively studied. Recently, we observed a graphitic structure (hereafter referred to as HX) in [0110]-orientated nanowires (Kulkarni et al., 2006) and a body-centered-tetragonal phase (hereafter referred to as BCT-4) in [0001]-oriented nanowires under uniaxial tensile loading (Wang et al., accepted for publication). Here, we characterize the phase transformations from WZ that lead to these novel structures. For comparison and overall perspective, the WZ-to-RS transformation is also analyzed. Our analyses use first-principles calculations based on the density functional theory (DFT) and molecular dynamics (MD) simulations. Particular interest is on the crystallographic changes and critical loading condition for each transformation. A crystalline structure-load triaxiality map is developed to summarize the relationship between the structures and load condition.

\section{Computational framework}

The MD simulations use the Buckingham potential with charge interactions (Binks and Grimes, 1993; Wolf et al., 1999). The calculations concern the quasi-static uniaxial tension of nanowires with the [0110] growth orientation and nanorods with the [0001] growth orientation and the hydrostatic compression of bulk $\mathrm{ZnO}$. The initial structures considered are single-crystalline and wurtzite-structured with lattice constants $a=3.249 \AA$ and $c=5.206 \AA$, as illustrated in Fig. 1(a) (Wang, 2004). The computational cell for bulk structure is $29.24 \times 28.13 \times 31.24 \AA$ in size and is created by repeating a unit wurtzite cell along the [00001], [01 10$]$ and $[2 \overline{1} 10]$ directions. Periodic boundary conditions (PBCs) are specified along the three directions to approximate infinite material extension. The [0110]-oriented nanowires have rectangular cross-sections and $\{2 \overline{1} \overline{1} 0\}$ and $\{0001\}$ lateral surfaces and the computational cell size is $21.22 \times 18.95 \times 150.83 \AA$. The [0001]-oriented nanorods have hexagonal cross-sections with a six-fold symmetry around the $[0001]$ axis and six $\{01 \overline{1} 0\}$ lateral surfaces. The corresponding computational cell size is $28.14 \times 65.0 \times 145.8 \AA$. PBCs are specified only along the axial directions for the nanowires.
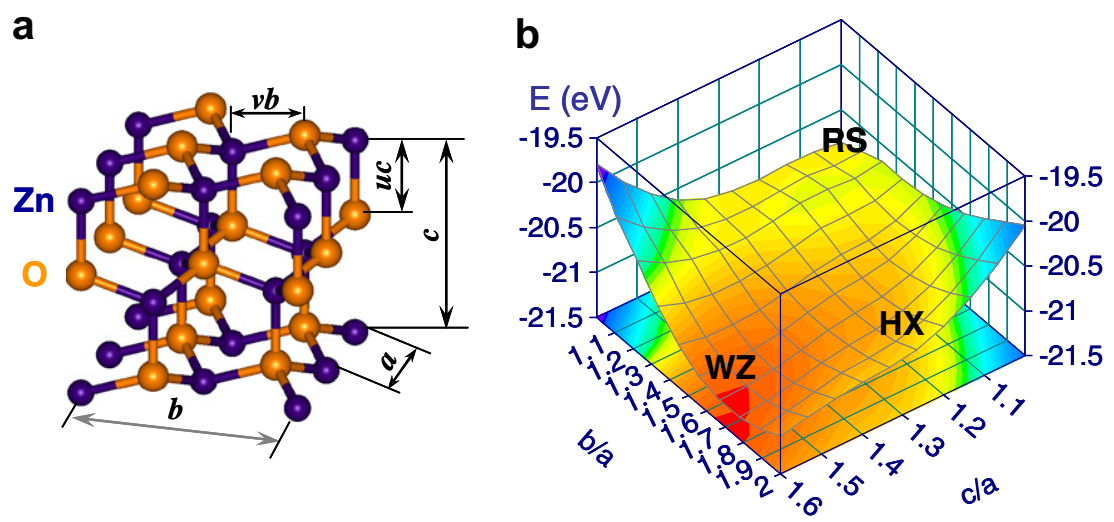

Fig. 1. (a) The wurtzite (WZ) crystal structure and (b) formation energy surface of ZnO with minima corresponding to the WZ, HX and RS structures. 
Since the crystallographically constructed computational cells may not be in equilibrium, pre-loading relaxations are carried out to obtain their free standing configurations. Following this initial equilibration, approximate quasi-static tensile loading in each deformation increment is achieved though successive loading (at a specified rate of $0.005 / \mathrm{ps}$ ) and equilibration steps (for $3 \mathrm{ps}$ ) using a combination of algorithms for NPT (Melchionna et al., 1993; Spearot et al., 2005) and NVE ensembles (Haile, 1997). The loading process results in a longitudinal strain increment of $0.25 \%$ (dilatation increment of $\sim 0.75 \%$ ) per deformation increment. Unloading is implemented in a similar manner with a reduction in strain for each unloading step. The virial formula is used to calculate the stress (Zhou, 2003).

The DFT calculations use the VASP code (Kresse and Furthmüller, 1996) with local density approximation (LDA) and ultrasoft pseudopotentials (Vanderbilt, 1990) and focus on the evaluation of the total energy in the natural and deformed states. The stability of each crystal structure is determined by analyzing enthalpy as a function of lattice parameter ratios $c / a$ and $b / a$. The enthalpy per unit cell ( $2 \mathrm{Zn}-\mathrm{O}$ pairs) under uniaxial loading is defined as

$$
H(c / a, b / a)=E(c, b, a, u, v)-\frac{1}{2} f_{i} q_{i},
$$

where $E$ is the formation energy, $f_{i}$ is the uniaxial force along the $i$ direction, $q_{i}$ is the lattice parameter in the $i$ direction, and $f_{i} q_{i}$ (summation not implied) is external work per unit volume $V$. For tension along the $[01 \overline{1} 0]$ axis, $i=b, f_{b}=\sigma_{b} \times(a c)$ and $q_{b}=b$, with $\sigma_{b}$ being the tensile stress. For tension along the $c$ axis, $i=c$, $f_{c}=\sigma_{c} \times(a b)$, and $q_{c}=c$, with $\sigma_{c}$ being the tensile stress. For hydrostatic compression, the enthalpy is

$$
H(c / a, b / a)=E(c, b, a, u, v)+\frac{1}{2} p V,
$$

where $p$ is the pressure and $V=a b c$ is the volume of two unit cells containing $4 \mathrm{Zn}-\mathrm{O}$ pairs. For each $c / a$ and $b / a$ pair, the internal parameters $u$ and $v$ and the volume $V$ are allowed to relax so that the configuration that yields minimum $H$ is obtained. For a given load condition, the minima on the enthalpy surface with $c / a$ and $b / a$ as the independent variables identify the corresponding stable and metastable structures.

\section{Results and discussion}

\subsection{Stress-free state}

Under ambient conditions, $\mathrm{ZnO}$ assumes the $\mathrm{WZ}$ structure which belongs to the $P 6_{3} m c$ space group. As shown in Fig. 1a, this structure consists of two hexagonal close packed sublattices (one for $\mathrm{Zn}$ and the other for $\mathrm{O}$ ) with an offset of ' $u c$ ' along the [0 001 1] axis. The lattice parameters $a, b, c, u=u c / c, v=v b / b$ which completely define the structure are also indicated in the figure. Fig. $1 \mathrm{~b}$ shows the formation energy (or enthalpy at zero external loading) landscape for $\mathrm{ZnO}$. The structures corresponding to $\mathrm{WZ}, \mathrm{RS}$ and $\mathrm{HX}$ are shown. The global minimum occurs at the WZ structure with $(c / a, b / a)=(1.61,1.73)$. Clearly, WZ is the most stable structure with the lowest energy; HX and RS have higher energies and are not stable under ambient conditions.

\subsection{Uniaxial tension along the $[01 \overline{1} 0]$ orientation}

Fig. 2a shows an intermediate configuration during the tensile loading of a [0110]-oriented nanowire with the cross-sectional size of $21.22 \times 18.95 \AA$ using MD simulations. The corresponding stress-strain $(\sigma-\varepsilon)$ response is shown in Fig. 2b. The region between A and B corresponds to elastic stretching of the WZ structure. Loading beyond $\mathrm{B}$ results in a stress drop from 10.02 to $6.98 \mathrm{GPa}(\mathrm{B} \rightarrow \mathrm{C})$ at $\varepsilon=5.14 \%$. This softening behavior corresponds to the nucleation of the HX phase. At this stage, $u$ changes from its initial value of 0.38 for WZ to a value of 0.5 for $\mathrm{HX}$, implying the flattening of the buckled wurtzite basal plane ( $\mathrm{Zn}$ and $\mathrm{O}$ atoms becoming co-planar) [Fig. 2c]. As a result, $\mathrm{Zn}$ atoms are at equal distances from $\mathrm{O}$ atoms along the [000 01$]$ axis and the structure acquires the additional symmetry of a mirror plane perpendicular to the [0001] axis. This process occurs while the orientation of the basal plane remains invariant. Since $v$ remains unchanged, HX has the same hexagonal symmetry around the $c$-axis as WZ. As the deformation progresses, the transformed 
a

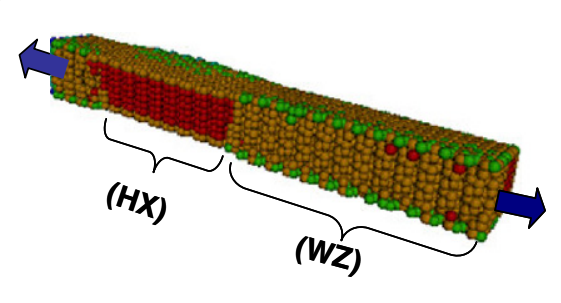

[0001]

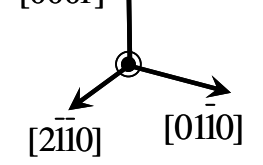

Coordination

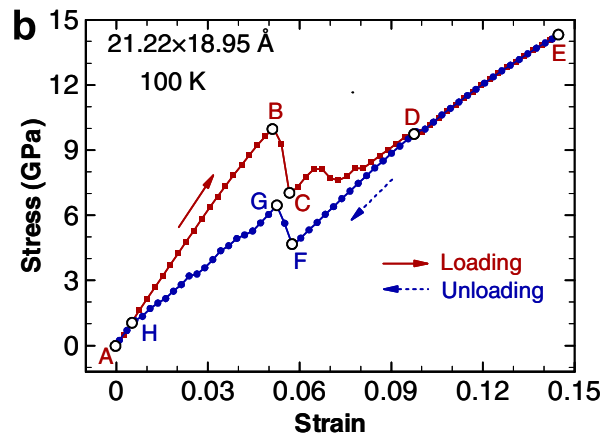

C

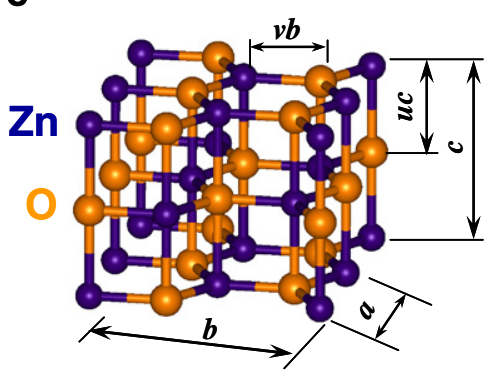

HX
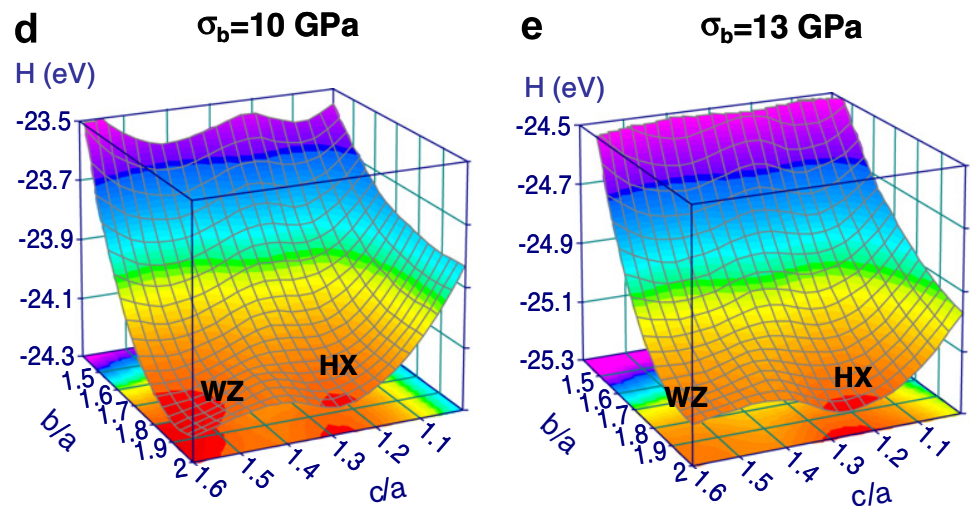

Fig. 2. (a) Nanowire with $\mathrm{HX}$ and $\mathrm{WZ}$ phases (transformation in progress under uniaxial tensile loading in the $[01 \overline{1} 0]$ ), (b) tensile stressstrain response of a $21.22 \times 18.95 \AA$ nanowire at $100 \mathrm{~K}$ during loading-unloading, (c) newly discovered hexagonal (HX) crystal structure, (d) enthalpy surface map obtained from DFT calculations with tensile stress $\sigma_{b}=10 \mathrm{GPa}$, and (e) enthalpy surface map with tensile stress $\sigma_{b}=13 \mathrm{GPa}$.

region sweeps through the entire wire length $(\mathrm{C} \rightarrow \mathrm{D})$ and the transformation completes at $\varepsilon=9.71 \%$ $(\sigma=9.65 \mathrm{GPa})$. Further deformation occurs through the elastic stretching of the transformed structure $(\mathrm{HX})$ and ultimate fracture occurs at $\varepsilon=16 \%(\sigma=15.29 \mathrm{GPa}$, not shown $)$ through cleavage along $\{\overline{1} 2 \overline{1} 0\}$ planes.

Unloading from any strain prior to the initiation of failure, e.g. point $\mathrm{E}$ with $\varepsilon=14.5 \%$, is first associated with the recovery of the elastic deformation within the $\mathrm{HX}$ structure $(\mathrm{E} \rightarrow \mathrm{F})$. A reverse transformation from $\mathrm{HX}$ to $\mathrm{WZ}(\mathrm{F} \rightarrow \mathrm{G} \rightarrow \mathrm{H})$ initiates at $\varepsilon=5.77 \%(\sigma=4.59 \mathrm{GPa}$, point $\mathrm{F})$ and completes at $\varepsilon=0.6 \%$ $(\sigma=1.15 \mathrm{GPa}$, point $\mathrm{H})$. Unloading beyond $\mathrm{H}$ occurs through elastic deformation within the WZ structure $(\mathrm{H} \rightarrow \mathrm{A})$. Strains up to $14.5 \%$ can be recovered, highlighting a very unusual aspect of the behavior of $\mathrm{ZnO}$ which normally is quite brittle. Obviously, the large recoverable strains observed here are associated with the unique structural transformation process. The energy dissipation associated with the stress-strain hysteresis loop is $\sim 0.16 \mathrm{GJ} / \mathrm{m}^{3}$, much lower than that for the WZ $\leftrightarrow \mathrm{RS}$ transformation in bulk $\left(\sim 1.38 \mathrm{GJ} / \mathrm{m}^{3}\right.$ with a maximum recoverable volumetric strain of $17 \%$ in compression) (Desgreniers, 1998).

Fig. $2 \mathrm{~d}$ and e shows the enthalpy surfaces (eV/unit cell) for $\sigma_{b}=10$ and $13 \mathrm{GPa}$, respectively. In each case, there are two minima. The first minimum $\left(H_{\min }^{\mathrm{WZ}}\right)$ is in the vicinity of $c / a \approx 1.6$ and $b / a \approx 1.9$ corresponding to a WZ structure with lattice parameters slightly different from those at zero stress. The second minimum $\left(H_{\min }^{\mathrm{HX}}\right)$ in the vicinity of $c / a \approx 1.3$ and $b / a \approx 1.9$ corresponds to the HX phase. At a stress value of $10 \mathrm{GPa}, H_{\min }^{\mathrm{HX}}$ and $H_{\min }^{\mathrm{WZ}}$ are comparable and consequently both WZ and $\mathrm{HX}$ are equally favored. At an applied stress of $13 \mathrm{GPa}$ [Fig. 3e], $H_{\mathrm{min}}^{\mathrm{HX}}$ is lower than $H_{\mathrm{min}}^{\mathrm{WZ}}$, indicating that $\mathrm{HX}$ is more stable. Obviously, the critical stress value for the WZ-to-HX transformation is $\sigma_{b} \approx 10 \mathrm{GPa}$. As the magnitude of $\sigma_{b}$ is increased above this equilibrium transition value, HX becomes more stable and simultaneously the transformation barrier is even lower, resulting in an increased driving force for transformation. 


\subsection{Uniaxial tension along the [0001] orientation}

Fig. 3a shows the configuration of a [0001]-oriented nanorod with a lateral dimension of $d=32.5 \AA$. Fig. $3 \mathrm{~b}$ shows the corresponding stress-strain response. Four distinct stages $(\mathrm{A} \rightarrow \mathrm{B}, \mathrm{B} \rightarrow \mathrm{C}, \mathrm{C} \rightarrow \mathrm{D}$ and $\mathrm{D} \rightarrow \mathrm{E})$ are observed. The first stage $(\mathrm{A} \rightarrow \mathrm{B})$ corresponds to the elastic stretching of the WZ structure up to a strain of $7.5 \%$. Further deformation results in a precipitous stress drop $(B \rightarrow C)$ associated with the WZ to BCT-4 phase transformation which initiates in a local zone and propagates along the length of the nanorod. Crystallographic analysis reveals that the transformed phase consists of four-atom ( $2 \mathrm{Zn}$ and $2 \mathrm{O}$ ) rings arranged in a BCT lattice [Fig. 3c]. The four-atom ring at the center has an orientation different (rotated by $90^{\circ}$ ) from that of the rings at the corners of the tetragonal lattice cell. The BCT-4 structure thus obtained preserves the initial tetrahedral coordination such that each $\mathrm{Zn} / \mathrm{O}$ atom is at the center and four $\mathrm{O} / \mathrm{Zn}$ atoms are at the vertices of a distorted tetrahedron. The distortion in the coordination tetrahedron can be analyzed through a quantification of the 3-D O-Zn-O bond angles $\left(\alpha_{i}, i=1 . .6\right)$. For WZ, the bond angles are approximately equal $\left(\alpha_{i} \approx 108^{\circ}\right)$. For BCT-4, the formation of 4-atom rings results in three distinct groups of bond angles $\left(\alpha_{1} \approx 90^{\circ}, \alpha_{2} \approx 112.7^{\circ}\right.$ and $\left.\alpha_{3} \approx 113.7^{\circ}\right)$. Throughout the transformation, the $b / a$ ratio remains at its initial value of $1.73( \pm 0.02)$, reflecting the symmetries of the loading and the lattice. The transformation completes at a strain of $8.5 \%$. Further loading causes the elastic stretching of the BCT-4 structure $(C \rightarrow D)$ and culminates in the eventual failure at a strain of $16.9 \%$ (point $\mathrm{E}$ ).

To analyze the stability of the WZ and BCT-4 structures, unloading is performed from points B and D which correspond, respectively, to the states prior to the transformation initiation and failure initiation of the nanorod. The unloading path from B coincides with the loading path, confirming that the deformation from $\mathrm{A}$ to $\mathrm{B}$ is indeed the elastic response of the $\mathrm{WZ}$ structured nanorod. Unloading from $\mathrm{D}$ also results in the elastic recovery of the stretched BCT-4 structure and continued unloading beyond the transformation completion strain (point $\mathrm{C}$ ) does not result in a reverse transformation back to WZ. Instead, the nanorod

a

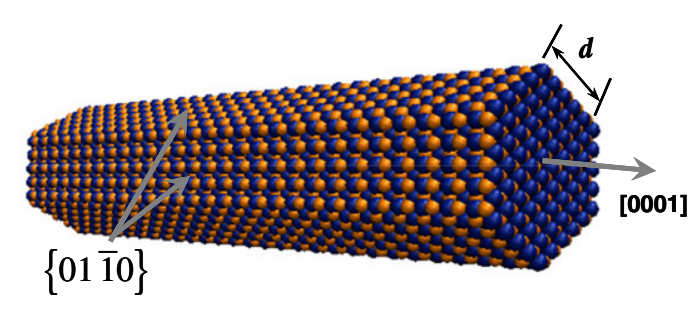

b

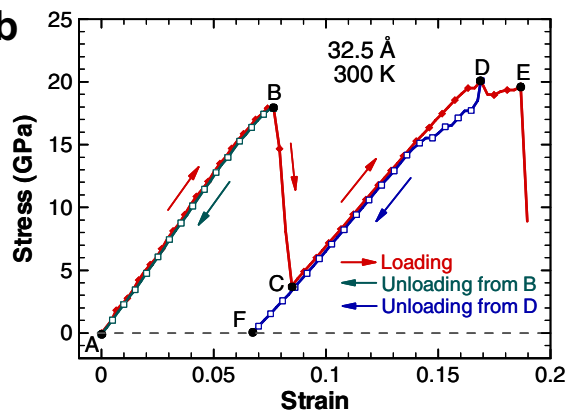

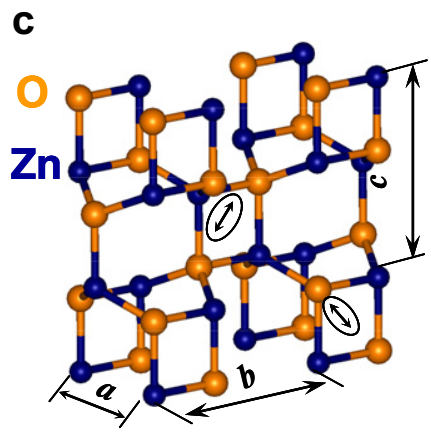

BCT- 4
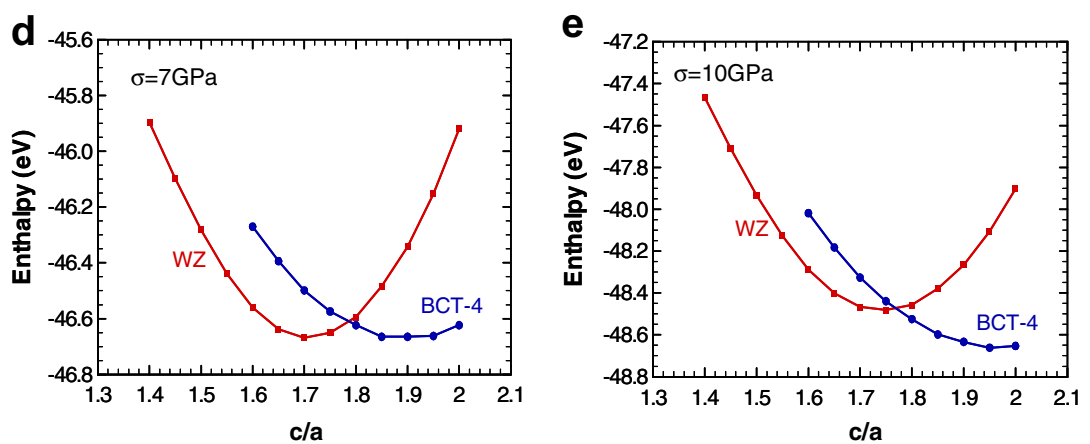

Fig. 3. (a) Configuration of a [0001] nanorod with $d=32.5 \AA$, (b) stress-strain curve of this nanorod at $300 \mathrm{~K}$ during loading and unloading, (c) newly discovered body-centered-tetragonal with four atom rings (BCT-4) structure, (d) enthalpy (per $4 \mathrm{Zn-O}$ pairs) as a function of $c / a$ obtained from DFT calculations for $b / a=1.73$ at a tensile stresses of $\sigma_{c}=7 \mathrm{GPa}$, and (e) enthalpy map at $\sigma_{c}=10 \mathrm{GPa}$. 
retains the BCT-4 structure when the stress is reduced to zero [F in Fig. 3b]. The residual strain at F in Fig. 3b is $6.8 \%$ according to both MD and DFT. It reflects the dimensional difference between the unstressed WZ and BCT-4 structures in the [0001] direction. This unstressed BCT-4 structure corresponds to the "ideal" BCT-4 structure predicted by the DFT calculations with $b / a=c / a=1.73$.

Fig. 3d and e show the enthalpy values (eV per $4 \mathrm{Zn}-\mathrm{O}$ pairs) for both $\mathrm{WZ}$ and BCT-4 with $b / a=1.73$ at $\sigma=7$ and $10 \mathrm{GPa}$. At any stress level, each structure has its own enthalpy minimum. The first minimum $\left(H_{\min }^{\mathrm{WZ}}\right)$ is in the vicinity of $c / a \approx 1.6$ which corresponds to a WZ structure with lattice parameters slightly different from those at zero stress and the second minimum $\left(H_{\min }^{\mathrm{BCT}-4}\right)$ is in the vicinity of $c / a \approx 1.7-1.9$ which corresponds to the BCT-4 structure. At a stress of $7 \mathrm{GPa}, H_{\min }^{\mathrm{WZ}}$ and $H_{\min }^{\mathrm{BCT}-4}$ become comparable, indicating that $\mathrm{WZ}$ and BCT-4 are equally favored. This value of stress corresponds to the equilibrium transition stress for the two phases. At $10 \mathrm{GPa}$ [Fig. 3c], $H_{\mathrm{min}}^{\mathrm{BCT}-4}$ is lower than $H_{\mathrm{min}}^{\mathrm{WZ}}$ and BCT-4 is clearly favored. Further increases in stress result in a higher driving force for and the eventual initiation of the phase transformation into the BCT-4 structure as $H_{\min }^{\mathrm{BCT}-4}$ becomes progressively lower than $H_{\mathrm{min}}^{\mathrm{WZ}}$. The gradual evolution of the local enthalpy minimum for the BCT-4 at $\sigma=0$ into a global minimum as stress increases confirms that the phase transformation is indeed favorable.

\subsection{Hydrostatic compression}

Fig. $4 \mathrm{a}$ and $\mathrm{b}$ show the initial $\mathrm{WZ}$ and transformed $\mathrm{RS}$ structures for $\mathrm{ZnO}$. The corresponding pressuredilatation relation is shown in Fig. 4c. Three distinctive stages of response during loading $(\mathrm{A} \rightarrow \mathrm{B}, \mathrm{B} \rightarrow \mathrm{C}$ and $\mathrm{C} \rightarrow \mathrm{D})$ and unloading $(\mathrm{D} \rightarrow \mathrm{E}, \mathrm{E} \rightarrow \mathrm{F}$ and $\mathrm{F} \rightarrow \mathrm{G})$ are observed. During loading, the first stage $(\mathrm{A} \rightarrow \mathrm{B})$ corresponds to the elastic deformation of the $\mathrm{WZ}$ structure. The precipitous drop in pressure at $p=9.4 \mathrm{GPa}$ is associated with the transformation of the initial WZ structure to the RS structure. Crystallographically, the
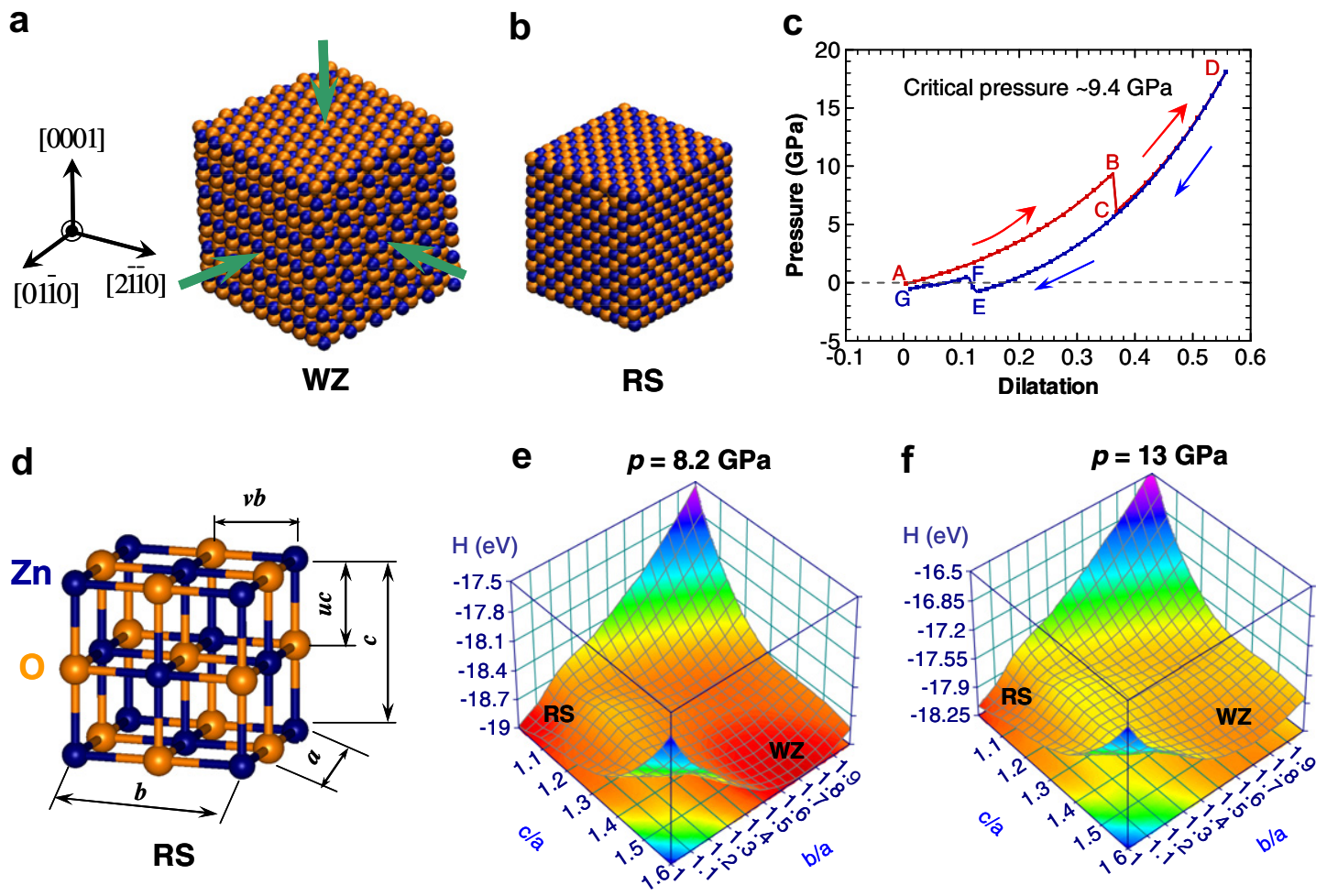

Fig. 4. (a) Bulk $\mathrm{ZnO}$ with the WZ structure under hydrostatic compression, (b) the RS structure as a result of the WZ-to-RS transformation, (c) pressure-dilatation relation of bulk $\mathrm{ZnO}$ during loading and unloading at $300 \mathrm{~K}$, (d) lattice structure of the RS phase, (e) enthalpy surface obtained from DFT calculations for a hydrostatic pressure of $p=8.22 \mathrm{GPa}$, and (f) enthalpy surface for $p=13 \mathrm{GPa}$. 


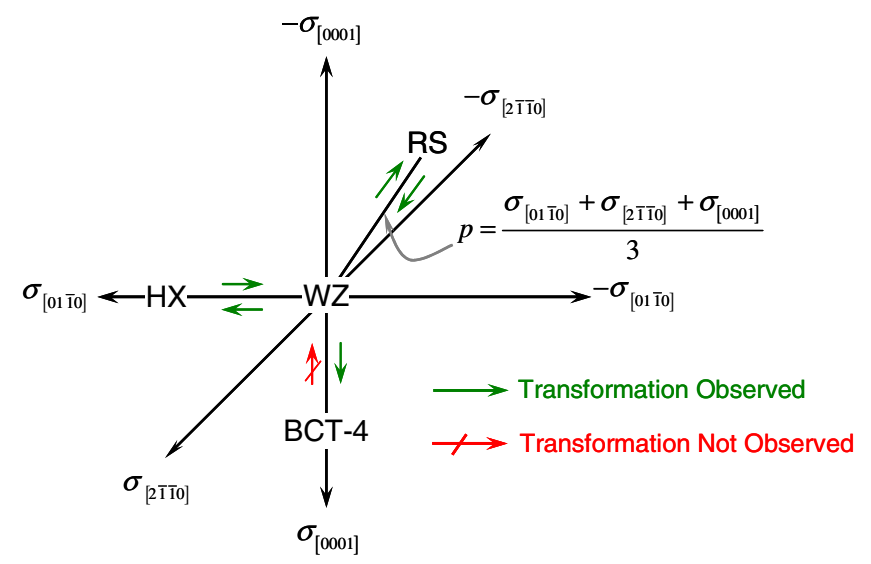

Fig. 5. Crystalline structure-load triaxiality map showing the relationship between applied loading and the resulting polymorphs of $\mathrm{ZnO}$.

transformation proceeds such that both $u$ and $v$ change to 0.5 and the $b / a$ and $c / a$ ratios become unity [Fig. 4d]. Consequently, the RS structure has a six-fold coordination and belongs to the $F m \overline{3} m$ space group. The transformation pressure observed here is consistent with experimental measurements in the range of 8.7-9.1 GPa (Desgreniers, 1998; Karzel et al., 1996). These values are higher than the phase equilibrium stress predicted by first-principles calculations (6.6-8.5 GPa) (Jaffe and Hess, 1993; Jaffe et al., 2000; Limpijumnong and Jungthawan, 2004). The difference is that, while the MD and experimental values are actual transformation stresses which reflect the effect of the energy barrier between the WZ and RS phases, the DFT phase equilibrium stress only indicates the level of stress at which the two phases are equally favored but does not relate to the stress required to overcome the energy barrier and activate the transformation.

Upon transformation completion, further increase in pressure results in the elastic deformation of the RS phase. Unloading is carried out from various stages of deformation of the RS phase to analyze the reversibility of the transformation. Specifically, unloading from point $\mathrm{D}$ along the loading path results in the recovery of the elastic deformation of the RS phase. Decrease in pressure beyond the transformation initiation point (B) does not result in the reverse transformation. Instead, the RS structure remains upon complete unloading. This retention of the RS structure upon full unloading has been reported in experiments (Recio et al., 1998). However, a spontaneous reverse transformation has also been observed in experiments (Mujica et al., 2003). Both sets of experimental results are reasonable because in experiments temperature is controlled only in an average sense and different experiments are carried out at different temperatures. Excess thermal energy in high temperature regions can allow the energy barrier between the RS and WZ structures to be overcome, resulting in the reverse RS-to-WZ transformation upon unloading. Furthermore, in experiments, defects such as grain boundaries in polycrystalline $\mathrm{ZnO}$ samples can act as potential nucleation sites for the reverse transformation and this effect is not considered in the calculations. In the simulations, the RS structure can easily revert to the $\mathrm{WZ}$ structure upon the application of a small negative hydrostatic pressure $(\mathrm{E} \rightarrow \mathrm{F})$, leading to full elastic recovery of the volumetric strain $(F \rightarrow G)$.

Fig. $4 \mathrm{e}$ and $\mathrm{f}$ show the enthalpy landscapes for $p=8.22$ and $13 \mathrm{GPa}$, respectively. Similar to what is seen for the $\mathrm{HX}$ and BCT-4 transformations, there are two minima; one corresponds to $\mathrm{WZ}\left(H_{\min }^{\mathrm{WZ}}, c / a \approx 1.6\right.$ and $b / a \approx 1.6)$ and the other corresponds to $\mathrm{RS}\left(H_{\min }^{\mathrm{RS}}, c l a \approx 1.0\right.$ and $\left.b / a \approx 1.0\right)$. At $p=8.22 \mathrm{GPa}, H_{\min }^{\mathrm{WZ}}=H_{\min }^{\mathrm{RS}}$ and $\mathrm{WZ}$ and $\mathrm{RS}$ are equally favored. This value of pressure is the phase equilibrium pressure for $\mathrm{ZnO}$. As the pressure is increased to $13 \mathrm{GPa}, H_{\min }^{\mathrm{RS}}$ becomes much lower than $H_{\mathrm{min}}^{\mathrm{WZ}}$ and the transformation to RS is energetically favored.

\section{Summary}

The identification of the novel HX and BCT-4 crystalline structures and the characterization of the WZ-toHX and WZ-to-BCT-4 phase transformations lead to a more complete understanding of the nature of 
polymorphism in $\mathrm{ZnO}$ and its dependence on load triaxiality. Obviously, polymorphism is much more pronounced in $\mathrm{ZnO}$ than previously understood and load triaxiality plays a very significant role in determining the structures. Fundamentally, this complexity is a reflection of the anisotropy and tension-compression asymmetry embedded in the atomic bonding and crystalline structures. It is possible to construct a crystalline structure-load triaxiality map for $\mathrm{ZnO}$, as shown in Fig. 5. Among the previously well known phases, WZ is the most stable and naturally occurring phase and RS is observed under hydrostatic compressive conditions. Both BCT-4 and HX are stabilized under uniaxial loading, with HX occurring under tension along the [01 10$]$ and BCT-4 occurring under tension along the [0 001 1] direction.

\section{Acknowledgement}

Support from NSF (CMS9984298) and NSFC (10772012, 10432050, and 10528205) is acknowledged. SL and KS are supported by TRF (BRG4880015 and PHD/0264/2545) and CHE (CHE-RES-RG "Theoretical Physics"). Computations are carried out at the NAVO, ARL, AHPCRC \& ASC MSRCs.

\section{References}

Binks, D.J., Grimes, R.W., 1993. Incorporation of monovalent ions in ZnO and their influence on varistor degradation. J. Amer. Cer. Soc. 76, 2370-2372.

Desgreniers, S., 1998. High-density phases of ZnO: Structural and compressive parameters. Phys. Rev. B 58, $14102-14105$.

Diao, J. et al., 2004. Surface stress driven reorientation of gold nanowires. Phys. Rev. B 70, 075413.

Haile, J.M., 1997. Molecular Dynamics Simulation. Wiley Interscience, New York.

Jaffe, J.E., Hess, A.C., 1993. Hartree-Fock study of phase changes in ZnO at high pressure. Phys. Rev. B 48, 7903-7909.

Jaffe, J.E. et al., 2000. LDA and CGA calculations for high-pressure phase transition in ZnO and MgO. Phys. Rev. B 62, 1660-1665.

Karzel, H. et al., 1996. Lattice dynamics and hyperfine interactions in ZnO and ZnSe at high external pressures. Phys. Rev. B 53, 1142511438.

Kresse, G., Furthmüller, J., 1996. Efficiency of ab initio total energy calculations for metals and semiconductors using a plane-wave basis set. Comp. Mat. Sci. 6, 15-50.

Kulkarni, A.J. et al., 2005. Orientation and size dependence of the elastic properties of ZnO nanobelts. Nanotechnology 16, $2749-2756$.

Kulkarni, A.J. et al., 2006. Novel phase transformation in ZnO nanowires under tensile loading. Phys. Rev. Lett. 97, 105501-105504, 105502.

Liang, W., Zhou, M., 2006. Atomistic simulations reveal shape memory of fcc metal nanowires. Phys. Rev. B 73, 115401-115411, 115409.

Limpijumnong, S., Jungthawan, S., 2004. First-principles study of the wurtzite-to-rocksalt homogeneous transformation in ZnO: A case of a low-transformation barrier. Phys. Rev. B 70, 054101-054104, 054104.

Melchionna, S. et al., 1993. Hoover NPT dynamics for systems varying in shape and size. Mol. Phys. 78, 533-544.

Mujica, A. et al., 2003. High-pressure phases of group-IV, III-V, and II-VI compounds. Rev. Mod. Phys. 75, 863.

Ozgur, U. et al., 2005. A comprehensive review of ZnO materials and devices. J. Appl. Phys. 98, 041301.

Recio, J.M. et al., 1998. Compressibility of the high-pressure rocksalt phase of ZnO. Phys. Rev. B 58, 8949-8954.

Spearot, D. et al., 2005. Nucleation of dislocations from [001] bicrystal interfaces in aluminum. Acta Mat. 53, 3579-3589.

Vanderbilt, D., 1990. Soft self-consistent pseudopotentials in a generalized eigenvalue formalism. Phys. Rev. B 41, 7892.

Wang, J. et al., accepted for publication. Molecular dynamics and density functional studies of a body-centered-tetragonal polymorph of ZnO. Phys. Rev. B.

Wang, Z.L., 2004. Zinc oxide nanostructures: growth, properties and applications. J. Phys.:Cond. Matt. 16, R829-R858.

Wolf, D. et al., 1999. Exact method for the simulation of Coulombic systems by spherically truncated, pairwise $r^{-1}$ summation. J. Chem. Phys. 110, 8254-8282.

Zhou, M., 2003. A new look at the atomic level virial stress: on continuum-molecular system equivalence. In: Proceedings of the Royal Society of London 459, 2347-2392. 